\title{
Priority-Based Inter-Vehicle Communication for Highway Safety Messaging Using IEEE 802.11e
}

\author{
Chakkaphong Suthaputchakun \\ Computer Engineering Department, Bangkok University, Phahonyothin Road, Pathum Thani 12120, Thailand \\ Correspondence should be addressed to Chakkaphong Suthaputchakun, chakkaphong.s@bu.ac.th
}

Received 21 November 2008; Accepted 9 May 2009

Recommended by Maode Ma

\begin{abstract}
We propose to use IEEE 802.11e in conjunction with retransmission mechanisms for priority-based Intervehicle Communication (IVC) for highway safety messaging in both vehicular infrastructure and ad hoc networks. Each IVC message, which is assigned a priority based on the safety event urgency, requires different quality of service in terms of communication reliability and average delay. To increase the communication reliability in a broadcast-based IVC, we apply retransmission mechanisms that can provide proportional reliability differentiation for each prioritized message. We evaluate the performance of our proposed protocol using OPNET Modeler, in terms of percentage of successful transmissions and average delay. The protocol performance is evaluated for (1) different system parameters such as the packet size, number of vehicles, and percentage of priority 1 vehicles, and (2) prioritybased protocol parameters, such as contention window, interframe spacing and retransmission parameters. The results show that proper protocol parameter settings per priority message result in an efficient solution for priority based broadcast IVC. Moreover, the proposed protocol is fully compatible with both IEEE 802.11 and IEEE 802.11e standards.
\end{abstract}

Copyright (C) 2009 Chakkaphong Suthaputchakun. This is an open access article distributed under the Creative Commons Attribution License, which permits unrestricted use, distribution, and reproduction in any medium, provided the original work is properly cited.

\section{Introduction}

Due to the growing number of vehicles over the last five decades, we have now a saturation of the transportation infrastructure. Therefore, traffic congestion, accidents and transportation delays become unavoidable [1-3]. For example, in European Union alone, there are more than 3.5 million injured people and more than 160 billion EURO in material loses reported each year [4]. Several measures are implemented in order to solve these problems, such as implementation of safety systems (e.g., safety belts and airbags) and construction of more and better roads and highways. However, it is clear that building more roads is a solution limited by the large monetary investment required and also the lack of available space, which is particularly pronounced in urban areas.

Because of these concerns, Intelligent Transportation Systems research is chartered to alleviate highway safety problems, that is, develop safety applications that lead to a significant reduction of critical traffic events. Examples of safety applications are accident notification, traffic congestion warning and traffic congestion avoidance. These applications utilize a variety of data obtained from the host vehicle, the road and surrounding vehicles. Such data includes braking data from the cars ahead, road conditions sensed by surrounding cars, and data from other sensors in a vehicle. It is predicted that the introduction of highway safety applications will lead to at least $20 \%$ decrease in the average number of accidents $[5,6]$. As stressed by many researchers in [7-10], intervehicle communication with or without a fixed infrastructure provides necessary support for the deployment of such safety applications and the realization of an Automated Highway System [11].

As IVC significance was also recognized by the Federal Communications Commission (FCC), it allocated a block of spectrum in the 5.850 to $5.925 \mathrm{GHz}$ band. A number of national projects specifically address IVC, such as DSRC (WAVE) working group [12], German FleetNet [13], NOW projects [14], and Japanese Internet-ITS project [15].

The safety messages' importance leads to different message priorities which pose their own particular requirements 
on the communication system in terms of communication reliability and average delay. For example, when an accident occurs, accident related messages will be generated. These messages must be transmitted immediately with high reliability and low delay. Therefore, these messages should have a higher-priority when compared with traffic congestion related messages created during a traffic congestion period.

Most of the papers related to media access control (MAC) protocol design for IVC [16-22] do not account for message priority. However, without consideration of such priorities, emergency messages can be delayed, blocked and dropped by lower-priority messages, which are not urgent and can wait for later transmission. There are a few papers [23-25] that consider priority of messages. In [24], the authors proposed priority CSMA (P-CSMA) and polling P-CSMA (PP-CSMA). However, the protocol is not compatible with both the IEEE 802.11 and IEEE 802.11e standards. In addition, it is well known that the polling mechanism is not robust to channel errors and dynamic network topologies.

In [23] the authors use IEEE 802.11e Enhanced Distributed Channel Access (EDCA) to provide a priority scheme for broadcast IVC in ad hoc networks. However, the authors do not consider the broadcast transmission reliability issues, that is, they assume that each broadcast message is transmitted only once. Also, in their simulation study they only consider a single vehicle carrying highest priority messages, a fact that can skew the performance results. In other words, the authors did not consider contention among high-priority messages. This unavoidable contention can lead to significant collisions, causing low communication reliability. In contrast, in this paper we explicitly deal with this issue since it is important to provide highest reliability to highest priority messages. In particular, to overcome the reliability problem, we use priority-based retransmissions, that is, the number of retransmissions is a function of the message priority level. Moreover, we study and compare both infrastructure and ad hoc-based networks for a wide array of system and protocol parameters.

We introduced a priority scheme for hierarchical military ad hoc networks in [25] and safety vehicular ad hoc networks in [26], however, in the paper, we compare the result of infrastructure with ad hoc-based networks, we have proposed before.

In this paper, we use broadcast-based IEEE 802.11e EDCA MAC protocol [27] in conjunction with retransmission mechanisms, for different network settings, that is, vehicular infrastructure and ad hoc networks. The proposed approach provides proportional service differentiation in both networks in terms of communication reliability and average delay. To increase the transmission reliability in broadcast based IVC, we employ different retransmission mechanisms. The retransmission parameters are uniquely set for each priority and each network setting. Higher-priority messages will be retransmitted more number of times than lower-priority messages, fact that results in higher reliability for higher-priority messages.

Our OPNET Modeler [28] based simulations reveal that the proposed protocol is a viable solution for priority-based IVC, that is, the protocol provides: (1) service differentiation for different message priorities, (2) very low average delays (significantly lower than the tolerated delays in IVC), and (3) high reliability (high percentage of successful transmissions), even when the number of vehicles is high.

The rest of the paper is organized as follows: Section 2 briefly discusses IEEE 802.11e standard and Section 3 introduces IVC safety message priorities. Detailed description of the proposed protocol and associated simulation results for both infrastructure and ad hoc vehicular networks are discussed in Sections 4 and 5, respectively. Section 6 compares the proposed protocol performance for both networks. Section 7 concludes the paper.

\section{IEEE 802.11e}

IEEE 802.11 legacy MAC does not support the concept of message differentiation based on different message priorities. Since the need to provide service differentiation between different traffic classes is eminent (e.g., provide differential service to traffic like voice, video and email), in July 1999, 802.11e task group was chartered to introduce Quality of Service (QoS) support and service differentiation for different types of applications such as background traffic, best effort traffic, video traffic, and voice traffic. IEEE 802.11e which was approved as a standard in July 2005 [27] defines two mechanisms, Enhanced Distributed Channel Access (EDCA) and Hybrid Coordination function controlled Channel Access (HCCA). EDCA considers three basic priority mechanisms for accessing the channel: contention window $(\mathrm{CW})$, backoff parameters $\left(\mathrm{CW}_{\min }\right.$ and $\left.\mathrm{CW}_{\max }\right)$, and idle time after which a transmission may occur (Arbitration Interframe Space, AIFS). Once a station accesses the channel, EDCA also provides the ability to differentiate the time interval for which a station is authorized to hold the channel (transmission opportunity, TXOP).

EDCA introduces the concept of access categories (ACs), each with its own queue. Each data packet that arrives from the higher layer and belongs to a specific application, is mapped into a corresponding AC. The mapping is done based on the application, that is, voice traffic, video traffic, background traffic and best effort traffic are mapped to access categories 3, 2, 1, and 0, respectively. Each access category $i$ uses contention parameters $\left(\mathrm{CW}_{\min }[i], \mathrm{CW}_{\max }[i]\right.$, AIFS[ $\left.i\right]$ and TXOP[i]). Basically, the smaller the values of $\mathrm{CW}_{\min }[i]$, $\mathrm{CW}_{\max }[i]$, and AIFS[i], the shorter the channel access delay for the corresponding $\mathrm{AC}$ and the higher the priority to acquire the medium. AIFS is computed as follows:

$$
\operatorname{AIFS}[i]=\operatorname{SIFS}+\operatorname{AIFSN}[i] \cdot \text { slot time, }
$$

where SIFS is Short Interframe Space and AIFSN[i] is an integer larger than 1. After sensing the medium idle for a time interval of AIFS[i], each AC calculates its own random backoff time $\left(\mathrm{CW}_{\min }[i] \leq\right.$ backoff time $\left.\leq \mathrm{CW}_{\max }[i]\right)$. The purpose of using different contention parameters for different queues is to give a low priority $\mathrm{AC}$ a longer waiting time than a high-priority $\mathrm{AC}$, so the high-priority $\mathrm{AC}$ is likely to access the medium earlier than the low priority AC. Note that the backoff times of different ACs are randomly 
TABLE 1: Examples of safety message priorities.

\begin{tabular}{lcc}
\hline Priority & Type & Examples \\
\hline Pri(1) & Accident & Air bag sensor \\
& & Vehicle's body sensor \\
Pri(2) & Possibility of accident & Thermal sensor \\
& & Hard break \\
Pri(3) & Warning & Surface condition \\
& & Road work warning \\
Pri(4) & General & Traffic congestion \\
& & Weather condition \\
\hline
\end{tabular}

generated and may reach zero simultaneously. This can cause an internal collision. In such a case, a virtual scheduler inside every node allows only the highest-priority AC to transmit first.

\section{IVC Message Priorities}

In this section we describe the concept of IVC message priorities and the internal queues in each vehicle.

Each message that arrives at the MAC from the higher layer is mapped into one priority. Examples of message priorities in highway safety messaging are shown in Table 1, however, numbers of priorities maybe difference depending upon requirements of each implementation.

We assume four internal queues per vehicle for the four different priority messages, instead of traffic types as in traditional 802.11e (see Figure 1). Each message will be queued in the queue according to its priority. There is a virtual collision handler, which handles the internal collision. The virtual collision handler will allow higher-priority messages to be transmitted before lower-priority messages. We adopt a non-preemptive policy. For each priority $i$ there are different values of the following parameters: $\mathrm{CW}_{\min }[i]$, $\mathrm{CW}_{\max }[i], \mathrm{AIFS}[i]$, and TXOP [i]. Therefore, higher-priority message will access the channel faster than lower-priority messages. Once the vehicle obtains the channel, it transmits only 1 MAC Service Data Unit (MSDU).

\section{Vehicular Infrastructure Network}

In this section, we discuss the unique characteristics of a vehicular infrastructure network, the proposed protocol and results obtained from the OPNET Modeler-based simulation.

4.1. Network Model. We assume a vehicular infrastructure network in which the intervehicle communication is controlled by Road Side Units (RSUs) [29-31] located on the side of the highway as shown in Figure 2. Each RSU acts as an access point that broadcasts all the messages received from one particular vehicle to all others in its communication range. Thus, every vehicle, which wishes to send messages, transmits the messages directly to the RSU using the EDCA protocol. Once the RSU receives a message, it rebroadcasts it immediately (the RSU does not

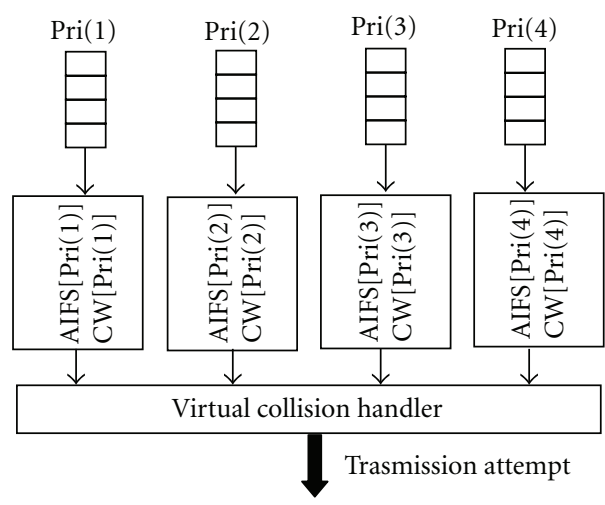

FIgURE 1: Vehicle queue architecture.

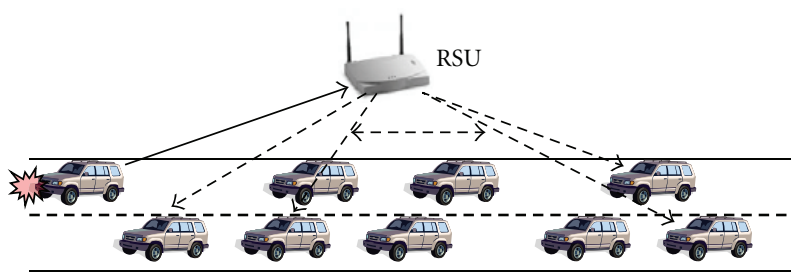

FIgURE 2: Vehicular infrastructure network.

compete with other vehicles for channel access since it has the highest priority RSU uses Point Interframe Space (PIFS), which is shorter than all values of AIFS[i]), to all other vehicles in range. As we briefly mentioned in the previous section, in conjunction with EDCA, different values of retry limits are assigned to different priorities ( $\mathrm{RL}[i]$ is assigned to priority $i$ messages). However, the actual number of retransmissions of each message may be lower than the retry limit, since the retransmission process will be terminated once the transmitting vehicle receives the acknowledgement back from the RSU. To increase the message reliability, the largest value of $\mathrm{RL}[i]$ must be assigned to the highest priority messages, thus providing more retransmission opportunities to the highest priority messages, increasing their probability of successful transmission.

4.2. Simulation Results and Analysis. To quantify the performance of our proposed protocol based on message priorities, we performed a simulation study using OPNET Modeler [28]. This section describes the simulation scenarios, configuration values and the performance in terms of percentage of successful transmissions (reliability) and average delay (this delay includes the queuing and transmission delays incurred by the message at the source vehicles and at the RSU) for each priority message.

We study the protocol performance as a function of (1) the protocol parameter in terms of retry limit for each priority message, $\mathrm{RL}[i]$, and (2) system parameters in terms of number of vehicles, $\mathrm{N}$, packet size, $\mathrm{L}$, and percentage of priority 1 vehicles, $\mathrm{P} 1$.

In our simulation we consider one RSU [31] and only two priorities, Pril and Pri4, in order to emphasize 


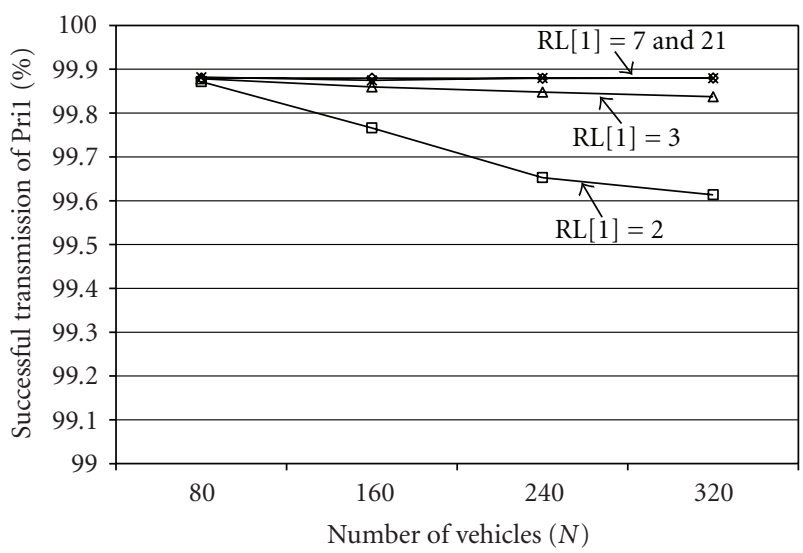

FIgure 3: Percentage of Pril successful transmissions as a function of $\mathrm{N}$ and RL[1] for a system described in Tables 2 and 3. Note: RL[4] $=1$.

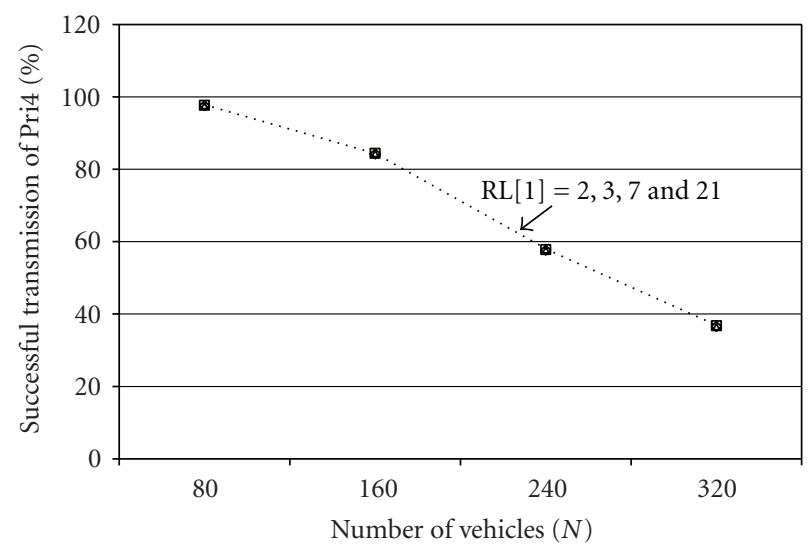

Figure 4: Percentage of Pri4 successful transmissions as a function of $\mathrm{N}$ and RL[1] for a system described in Tables 2 and 3. Note: RL[4] $=1$.

impact of different priorities. Each vehicle generates Poisson traffic $[21,22,32]$ with exponentially distributed packet interarrival time with mean $0.1 \mathrm{sec}$. The default packet size is 100 Bytes. Due to high speed vehicles and dynamic topology, the channel condition should not be as good as in regular wireless network. In all simulations, we decide to set bit error rate (BER) to $10^{-6} \mathrm{~dB}$ as the default channel condition. Table 2 shows all other default parameters used in the simulation. The default parameters for each priority $\mathrm{CW}_{\min }[i], \mathrm{CW}_{\max }[i], \operatorname{AIFSN}[i], \operatorname{RL}[i]$, and $\operatorname{TXOP}[i]$, are provided in Table 3.

4.2.1. Pri1 Retry Limit. In this subsection, we try to calibrate and find out the optimal value of the retry limit of Pril, $\mathrm{RL}[1]$, and study its impact on our system performance metrics, which are the percentage of successful transmissions and average delay, for both Pril and Pri4 messages. To observe the impact of RL[1], we use RL[4] = 1 and keep varying RL[1] from 2 to 21 . All other parameter values are provided in Tables 2 and 3.

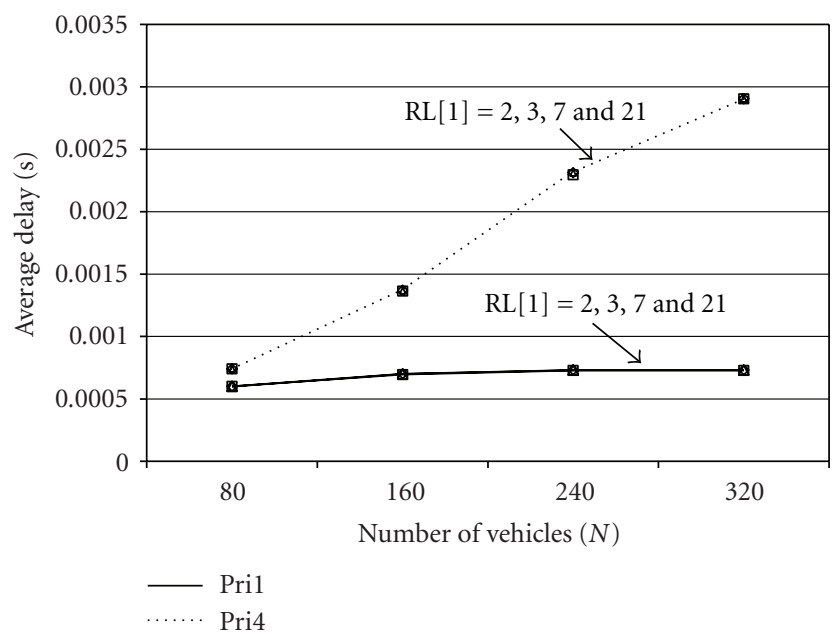

FIGURE 5: Average delay for each priority as a function of $\mathrm{N}$ and $\mathrm{RL}[1]$ for a system described in Tables 2 and 3. Note: $\mathrm{RL}[4]=1$.

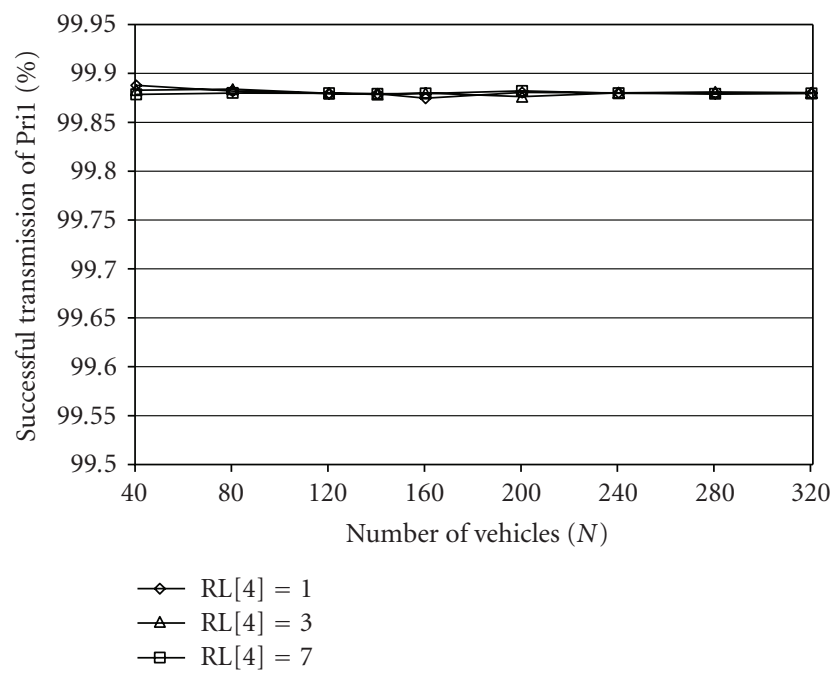

Figure 6: Percentage of Pril successful transmissions as a function of N and RL[4] for a system described in Tables 2 and 3. Note: RL[4] $=1$.

Figures 3 and 4 depict the percentage of successful transmissions of Pril and Pri4 messages, respectively, versus the number of vehicles, $\mathrm{N}$, for RL[1] from 2 to 21 . As shown in Figure 3, we observe that higher the RL[1], higher the reliability for Pril messages. However, we observe that at $\mathrm{RL}[1]=7$ we obtain the optimal performance in terms of percentage of successful transmissions. Further increase in the value of RL[1] does not improve the system performance because each message has never been retransmitted more than 7 times. In contrast, RL[1] does not have significant impact on the reliability of Pri4 messages as shown in Figure 4, since the percentage of successful transmissions of Pri4 messages is mainly based on RL[4], which is fixed at 1 .

Consequently, in the next experiments we will use RL[1] $=7$ as the default retry limit for Pril messages (see Table 3 ). 
TABLE 2: Default parameter values.

\begin{tabular}{lc}
\hline Parameters & Values \\
\hline Priorities of vehicles & 1 and 4 \\
Percentage of Pril vehicles & $5 \%$ \\
Percentage of Pri4 vehicles & $95 \%$ \\
Network interface & IEEE802.11a \\
Transmission range (outdoor) & $371 \mathrm{~m}$. \\
Simulation area & $10 * 10 \mathrm{Km}^{2}$ \\
Packet payload size & 100 Bytes \\
Packet generation interval & 0.1 second \\
Communication data rate & $6 \mathrm{Mbps}$ \\
Communication channel & Channel 1 \\
Bit error rate (BER) & $10^{-6} \mathrm{~dB}$ \\
Buffer size & 256,000 bits \\
Request to send (RTS) threshold & 256 Bytes \\
Fragmentation threshold & 2,034 Bytes \\
\hline
\end{tabular}

TABLE 3: Priority parameters.

\begin{tabular}{lccccc}
\hline Priority & $\mathrm{CW}_{\min }$ & $\mathrm{CW}_{\max }$ & AIFSN & RL & TXOP \\
\hline $\operatorname{Pri}(1)$ & $\mathrm{CW}_{\min / 4}$ & $\mathrm{CW}_{\min / 2}$ & 2 & 7 & $1 \mathrm{MSDU}$ \\
$\operatorname{Pri}(4)$ & $\mathrm{CW}_{\min }$ & $\mathrm{CW}_{\max }$ & 7 & 1 & $1 \mathrm{MSDU}$ \\
\hline
\end{tabular}

Figure 5 depicts the average delay versus $\mathrm{N}$ for different priorities and different values of RL[1]. From Figure 5, we notice that increasing RL[1] does not cause a significant impact on the average delay of both priority messages. This is due to the fact that the percentage of priority 1 messages is low; this is reflected in the relatively small increase in the traffic load which occurs when RL[1] increases.

Furthermore, from Figures 3, 4 and 5, we notice that for different values of RL[1], Pri1 messages gain higher reliability and lower delay compared with Pri4 messages due to the priority scheme we have used. We also observe that as the number of vehicles, $\mathrm{N}$, increases, the percentage of successful transmissions decreases and the average delay increases. This is due to the fact that as $\mathrm{N}$ increases, the traffic intensity in the network increases, increasing the number of collisions, decreasing the percentage of successful transmissions and also increasing the average delay of both priority messages.

4.2.2. Pri4 Retry Limit. In this subsection we investigate the impact of retry limit of Pri4, RL[4], on the system performance. Figures 6 and 7 depict the percentage of successful transmissions for Pril and Pri4, respectively, versus $\mathrm{N}$ for different values of RL[4]. Figure 8 depicts the average delay for both priority messages versus $\mathrm{N}$ for different values of RL[4]. As expected, we notice from Figures 6 and 8 that RL[4] does not cause a significant impact on the performance of Pril messages in terms of both communication reliability and average delay. In contrast, from Figures 7 and 8 we notice that RL[4] has a significant impact on the performance of Pri4 messages in terms of percentage of successful transmissions as well as the average delay. The higher RL[4], the lower Pri4 reliability and the

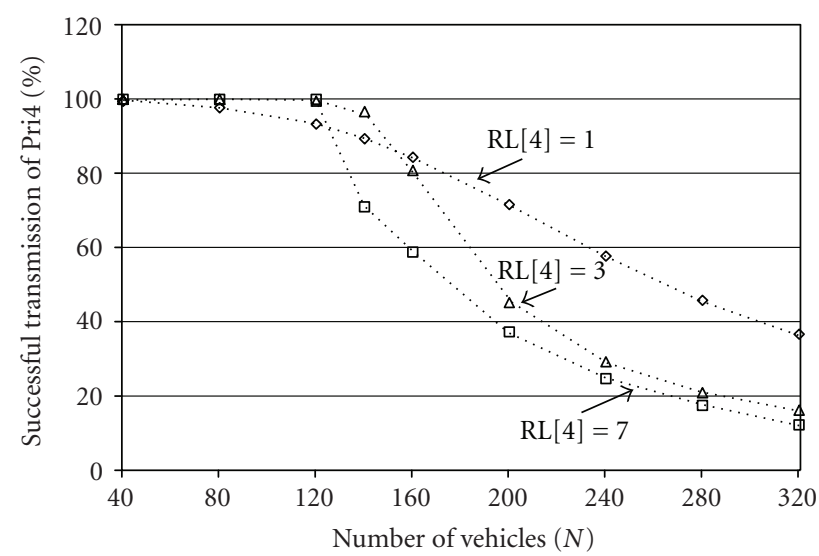

Figure 7: Percentage of Pri4 successful transmissions as a function of $\mathrm{N}$ and RL[4] for a system described in Tables 2 and 3. Note: RL[4] $=1$.

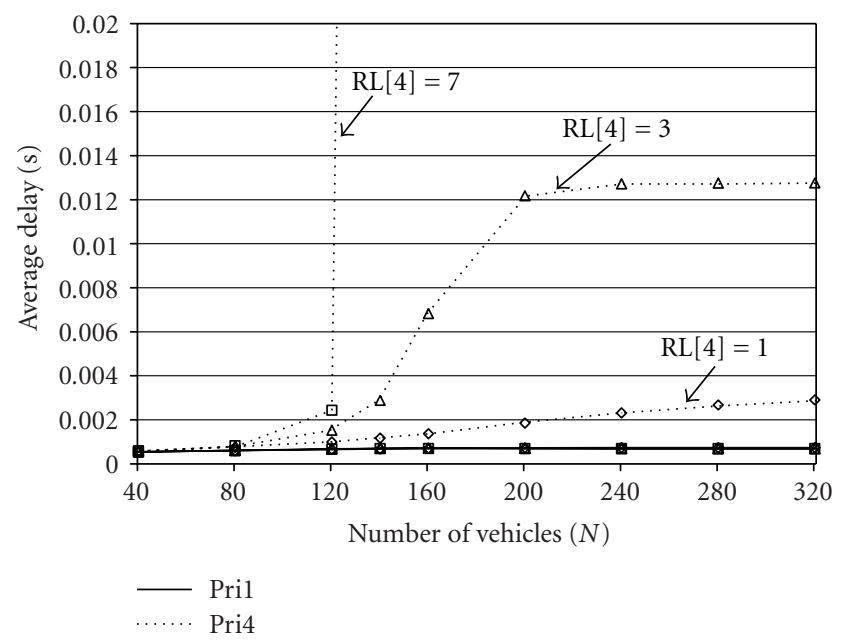

Figure 8: Average delay for each priority as a function of $\mathrm{N}$ and $\mathrm{RL}[4]$ for a system described in Tables 2 and 3. Note: RL[4] $=1$.

longer Pri4 delay. This fact is especially pronounced for large values of $\mathrm{N}$. Therefore, for the rest of the experiments we decide to use $\mathrm{RL}[4]=1$ as the default value.

4.2.3. Packet Size. We investigate the impact of the packet size, L, on the system performance. Figures 9 and 10 depict the percentage of successful transmissions versus $\mathrm{N}$ for Pri1 and Pri4, respectively, for different values of L. As shown in Figures 9 and 10, L impacts the reliability of both priority messages. Larger values of $\mathrm{L}$ cause higher traffic intensity, increasing the probability of collisions, and decreasing the percentage of successful transmissions. We also observe form both Figures 9 and 10 that the impact of L is very significant for Pri4 messages, while for Pril messages we observe only a slight degradation in their reliability.

Figure 11 depicts the average delay versus $\mathrm{N}$ for different priorities and different values of L. Consistent with the observations from Figures 9 and 10, from Figure 11 we observe that larger the values of $\mathrm{L}$, higher the delay for both 


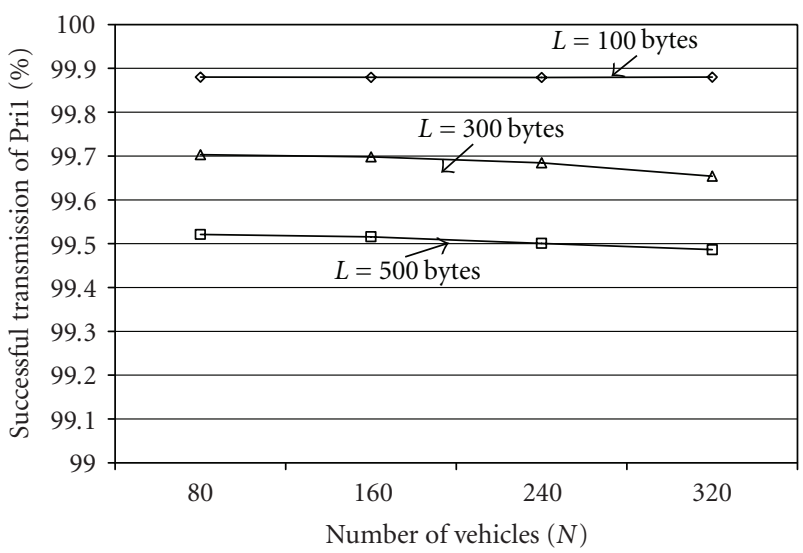

Figure 9: Percentage of Pril successful transmissions as a function of $\mathrm{N}$ and $\mathrm{L}$ for a system described in Tables 2 and 3.

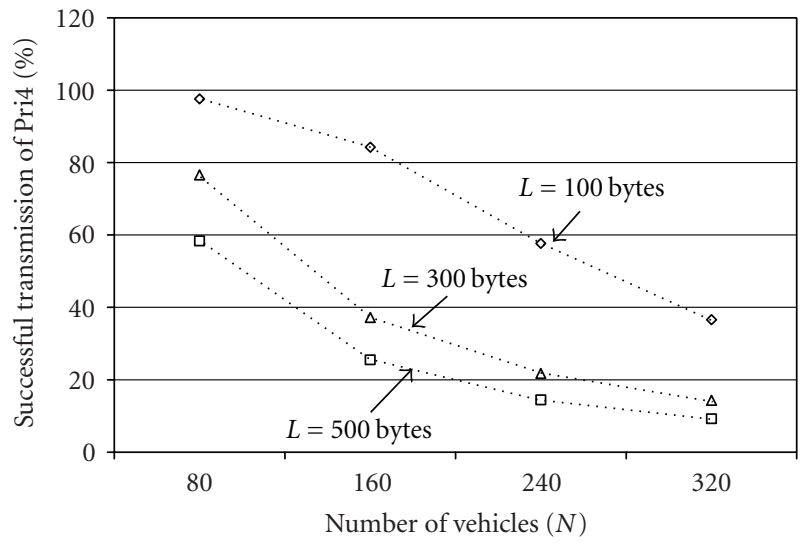

Figure 10: Percentage of Pri4 successful transmissions as a function of $\mathrm{N}$ and $\mathrm{L}$ for a system described in Tables 2 and 3.

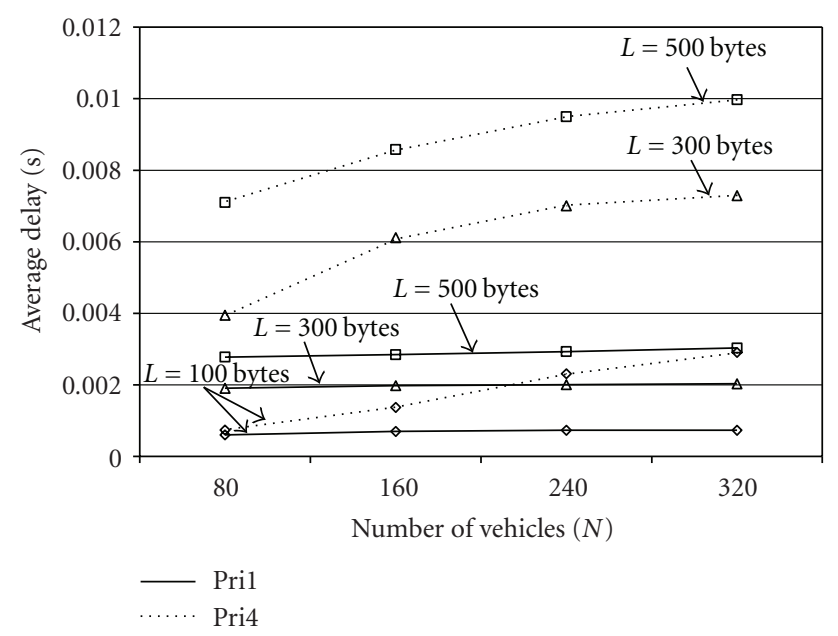

FIGURE 11: Average delay for each priority as a function of $\mathrm{N}$ and $\mathrm{L}$ for a system described in Tables 2 and 3.

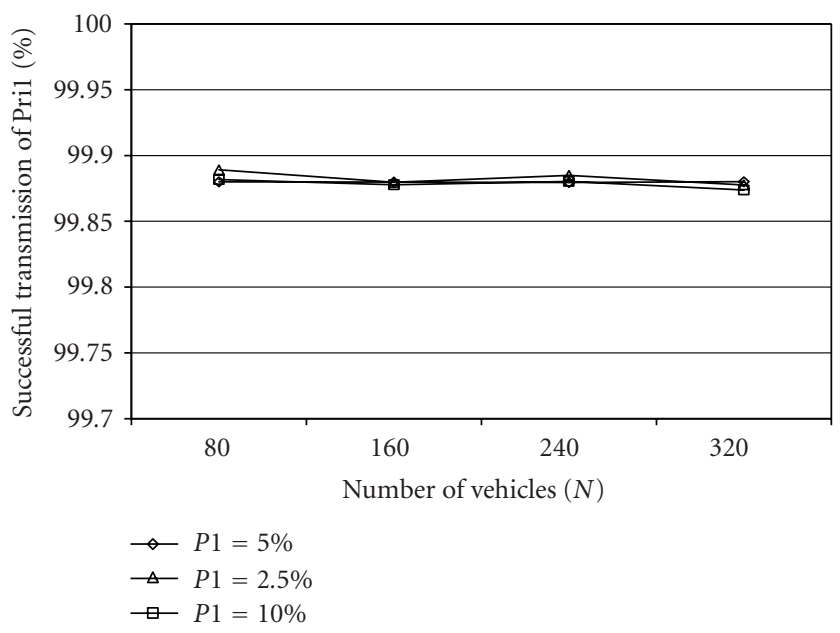

Figure 12: Percentage of Pril successful transmissions as a function of $\mathrm{N}$ and $\mathrm{P} 1$ for a system described in Tables 2 and 3.

priority messages. However, we notice that the average delay is still acceptable, that is, it is lower than the default lifetime of messages equal to $0.1 \mathrm{sec}$ [19], (default lifetime is the interval of time during which safety messages are useful).

4.2.4. Percentage of Pril Vehicles. The percentage of Pril messages, P1, depends on the traffic congestion, which is a function of the road setting (e.g., urban or rural areas) and the time of day (e.g., rush hours). For example, during rush hours in an urban area when traffic congestion is very high, it is very likely that the percentage of Pril messages is higher than that in rural areas during afternoon hours. As we will see in this subsection, P1 can significantly affect the IVC reliability.

To study the effect of $\mathrm{P} 1$ on both the system reliability and average delay in the vehicular infrastructure networks, we perform simulations for varying values of $\mathrm{P} 1, \mathrm{P} 1=2.5 \%$, $5 \%$, and $10 \%$. All other default parameter values are shown in Tables 2 and 3. Figures 12 and 13 depict the percentage of successful transmissions for Pril and Pri4, respectively, versus $\mathrm{N}$ for different values of P1. Figure 14 depicts the average delay for each priority versus $\mathrm{N}$ for different values of P1.

We observe from Figures 12 and 14 that Pri1 reliability and average delay remain almost constant regardless of the values of $\mathrm{N}$ and $\mathrm{P} 1$. In contrast, we notice from Figures 13 and 14 that, as $\mathrm{N}$ increases, Pri4 performance degradation is more pronounced. This is due to the fact that as $\mathrm{N}$ increases and P1 increases, Pri4 traffic intensity impacts the number of collisions resulting in decreased reliability and increased average delay.

\section{Vehicular Ad hoc Network}

In this section, we turn our attention to IVC in a vehicular ad hoc network. We describe the unique characteristics of the vehicular ad hoc network, the proposed protocol and simulation results obtained from OPNET Modeler. 
TABLE 4: Priority parameters.

\begin{tabular}{lccccc}
\hline Priority & $\mathrm{CW}_{\min }$ & $\mathrm{CW}_{\max }$ & AIFSN & NR & TXOP \\
\hline $\operatorname{Pri}(1)$ & $\mathrm{CW}_{\min / 4}$ & $\mathrm{CW}_{\max / 2}$ & 2 & 3 & $1 \mathrm{MSDU}$ \\
$\operatorname{Pri}(4)$ & $\mathrm{CW}_{\min }$ & $\mathrm{CW}_{\max }$ & 7 & 1 & 1 MSDU \\
\hline
\end{tabular}

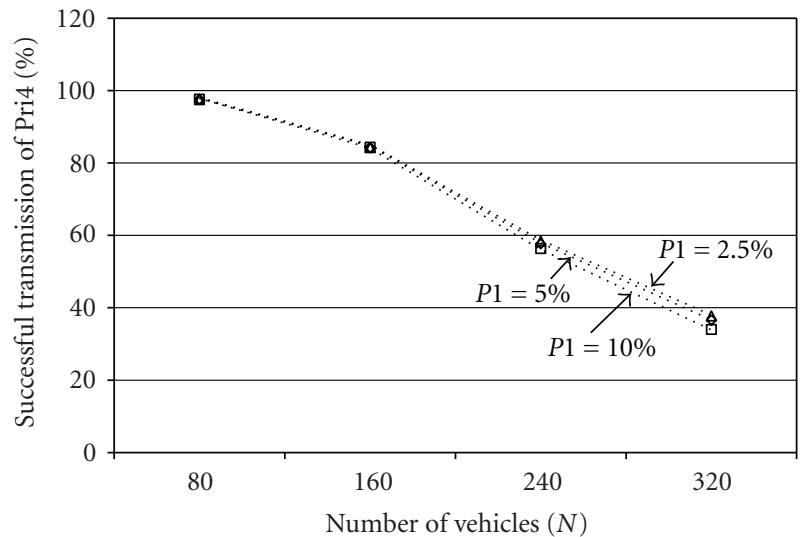

FIGURE 13: Percentage of Pri4 successful transmissions as a function of $\mathrm{N}$ and $\mathrm{P} 1$ for a system described in Tables 2 and 3.

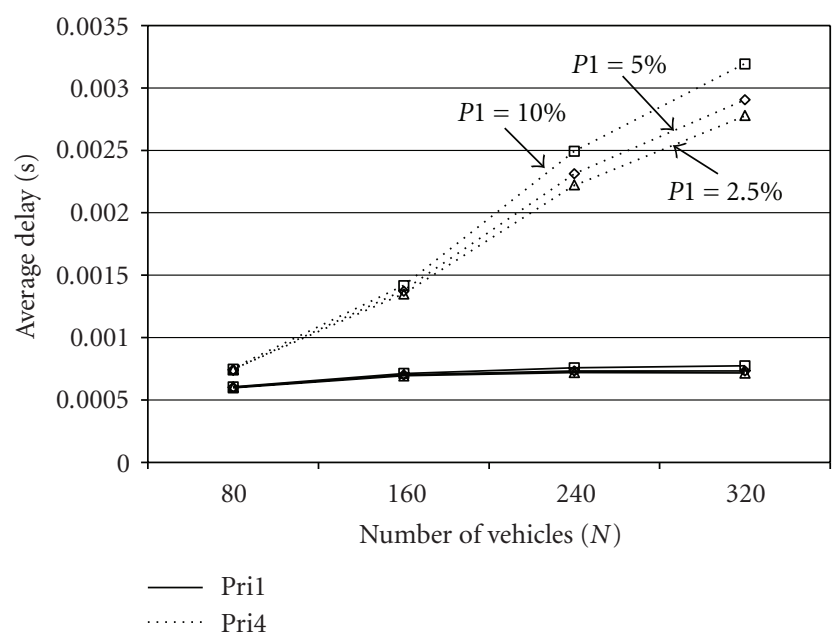

FIGURE 14: Average delay for each priority as a function of $\mathrm{N}$ and P1 for a system described in Tables 2 and 3.

5.1. Network Model. Due to the fact that RSU may not be provided in all segments of roads and highways, in this section, we focus on providing differential service to different priority messages in vehicular ad hoc networks as shown in Figure 15. In this network, since there is no infrastructure assistance, each vehicle has to rely on its own for communication, that is, it has to broadcast messages using EDCA and the priority scheme. Thus, there is no acknowledgement mechanism in ad hoc network unlike the infrastructure where acknowledgement is generated by RSU. Therefore, in ad hoc networks, one-time broadcasting can cause very low communication reliability.

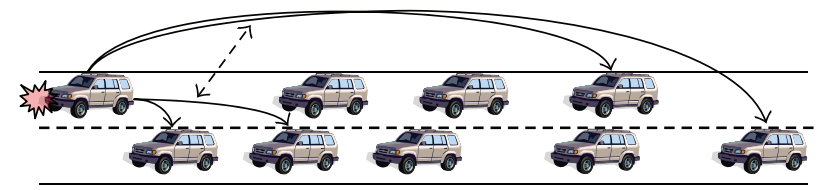

Figure 15: Vehicular Ad hoc network.

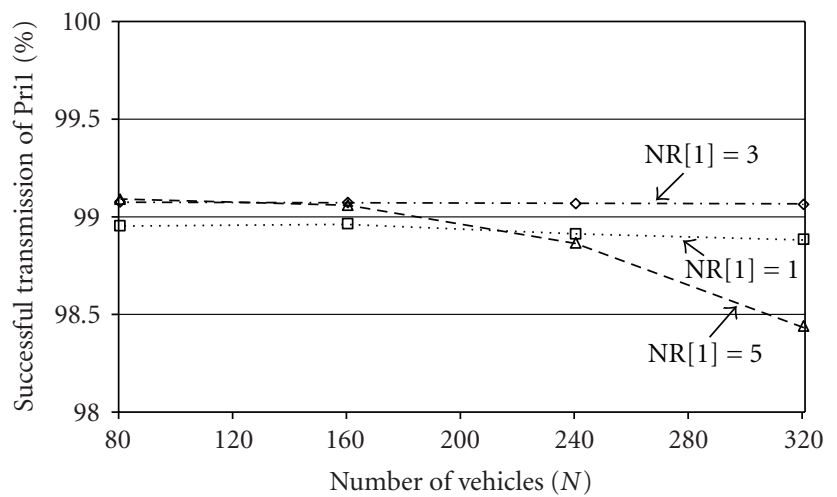

Figure 16: Percentage of Pril successful transmissions as a function of $\mathrm{N}$ and $\mathrm{NR}[1]$ for a system described in Tables 2 and 4. Note: $\mathrm{NR}[4]=1$.

In order to alleviate this issue, each vehicle will retransmit each message multiple times. The number of retransmissions is denoted by NR[i] (number of retransmissions for priority $i$ messages). To increase the probability of successful transmissions for higher-priority messages, they are assigned a higher $\mathrm{NR}[i]$.

5.2. Simulation Results and Analysis. This section describes the simulation scenario, configuration values and the performance in terms of average delay and percentage of successful transmissions for each priority.

We study the impact of different parameters on the system performance for two priorities, Pril and Pri4. We investigate the performance of our protocol as a function of number of retransmissions of each priority, NR $[i]$, number of vehicles, $\mathrm{N}$, packet size, $\mathrm{L}$, and percentage of priority 1 vehicles, $\mathrm{P} 1$.

Table 2 summarizes all default parameter values used in the simulation. The default parameters for each priority value $\mathrm{CW}_{\min }[i], \mathrm{CW}_{\max }[i], \operatorname{AIFSN}[i], \mathrm{TXOP}[i]$ and $\mathrm{NR}[i]$ are provided in Table 4.

5.2.1. Number of Retransmissions of Pri1. We investigate the impact of NR[1] on our performance metrics for both priority messages. We use $\mathrm{NR}[4]=1$ and $\mathrm{NR}[1]=1,3$, and 5. 


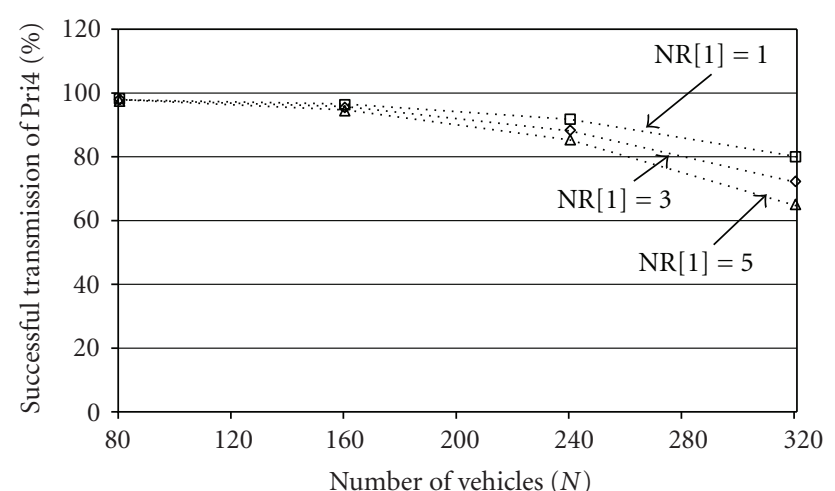

Figure 17: Percentage of Pri4 successful transmissions as a function of $\mathrm{N}$ and NR[1] for a system described in Tables 2 and 4. Note: $\mathrm{NR}[4]=1$.

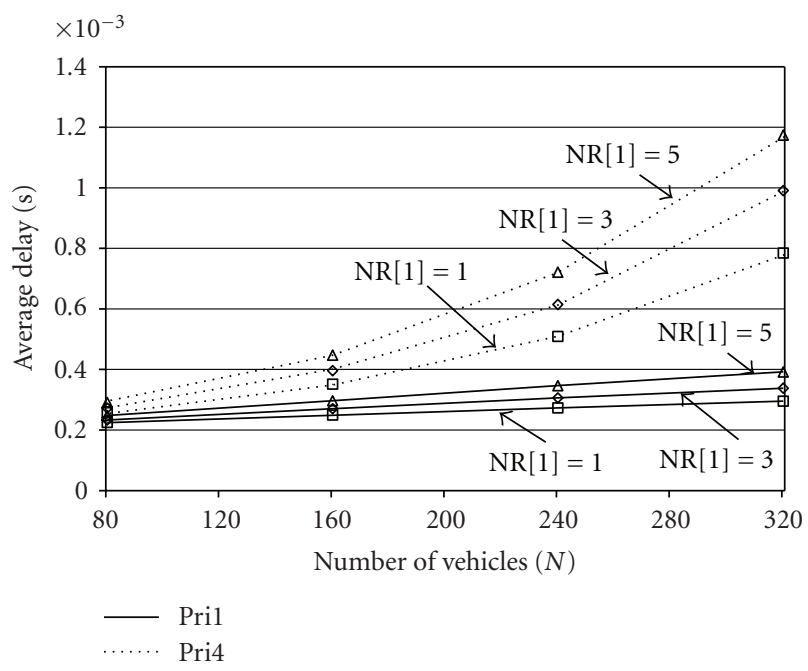

FIGURE 18: Average delay for each priority as a function of $\mathrm{N}$ and $\mathrm{NR}[1]$ for a system described in Tables 2 and 4 . Note: $\mathrm{NR}[4]=1$.

Figures 16 and 17 depict the percentage of successful transmissions versus N, for Pril and Pri4 messages, for different values of NR[1].

From Figure 16 we observe that there is an optimal value of NR[1]. As we increase NR[1] from 1 to 3, Pril reliability improves. However, as we keep increasing NR[1] to 5, Pri1 reliability deteriorates especially at large number of vehicles. This is due to the following reasons. We increase NR[1] from 1 to 3 , that is, increase the number of Pril messages 3 fold, but this is required to be able to get the messages through, that is, to increase the system reliability. However, further increase to $\mathrm{NR}[1]=5$, increases the number of messages 5 fold causing intense collisions and defeats the purpose, thus decreasing Pril reliability.

Consequently, we decide to use $\mathrm{NR}[1]=3$ as the default value, as shown in Table 4.

As observed from Figure 17, the higher NR[1] the lower the percentage of Pri4 successful transmissions. This is due to the fact that as NR[1] increases the traffic intensity of Pri1

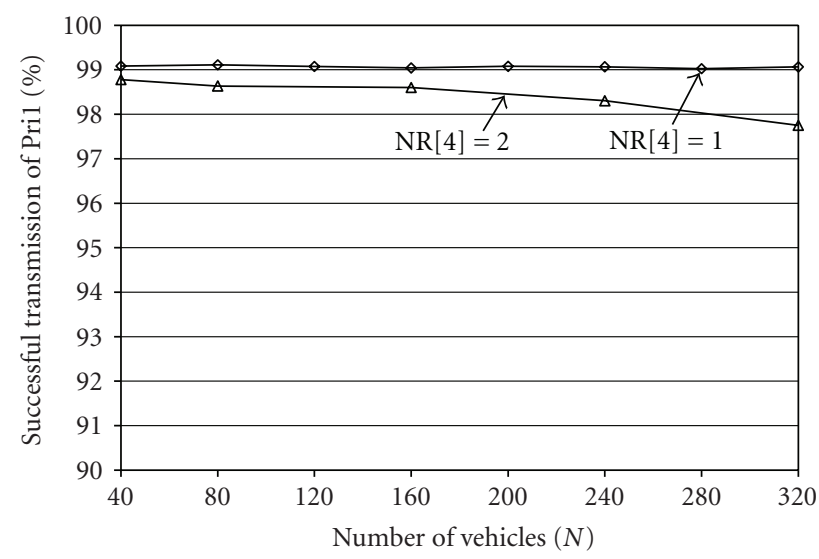

Figure 19: Percentage of Pril successful transmissions as a function of $\mathrm{N}$ and NR[4] for a system described in Tables 2 and 4 .

messages increases, leaving less bandwidth for Pri4 messages, decreasing Pri4 reliability.

Figure 18 depicts the average delay versus $\mathrm{N}$ for different priorities and different values of NR[1]. As expected, larger values of NR[1] cause higher delays for both priority messages. However, the average delay of both priority messages is still lower than the messages' default lifetime.

Furthermore, from Figures 16, 17 and 18, we also observe that a larger number of vehicles (i.e., increase traffic intensity and increase number of collisions) cause lower system reliability and higher average delay.

5.2.2. Number of Retransmissions of Pri4. In this subsection, we study the impact of NR[4] on the system performance. We use NR[1] = 3 (as concluded in the previous subsection) and $\mathrm{NR}[4]=1$, and 2 .

Figures 19 and 20 depict the percentage of successful transmissions versus $\mathrm{N}$ for Pril and Pri4 messages, respectively, for different values of NR[4]. We observe that as NR[4] increases from 1 to 2 there is a significant degradation in the percentage of successful transmissions. This is due to the fact that as NR[4] increases, the traffic intensity of Pri4 messages increases significantly (recall that $95 \%$ of the traffic includes Pri4 messages), increasing the potential collisions, thus decreasing the percentage of successful transmissions of both priority messages.

Figure 21 shows the average delay versus $\mathrm{N}$ for different priorities and different values of NR[4]. Consistent with our observations concluded from Figures 19 and 20, we notice that higher values of NR[4] increase the average delay for both priority messages.

From these results, we decide to use NR[4] $=1$ for the rest of the simulations.

5.2.3. Packet Size. In this subsection we focus our investigation on the impact of the packet size, L, on the system performance. Figures 22 and 23 depict the percentage of successful transmissions versus $\mathrm{N}$ for Pril and Pri4 messages, respectively, for different values of $\mathrm{L}$. We observe that increase in L causes a significant degradation of the system 


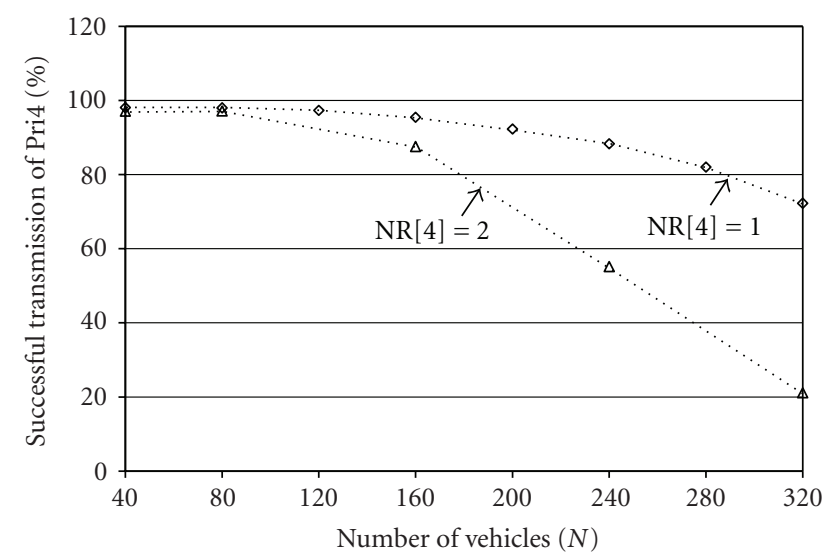

Figure 20: Percentage of Pri4 successful transmissions as a function of $\mathrm{N}$ and NR[4] for a system described in Tables 2 and 4.

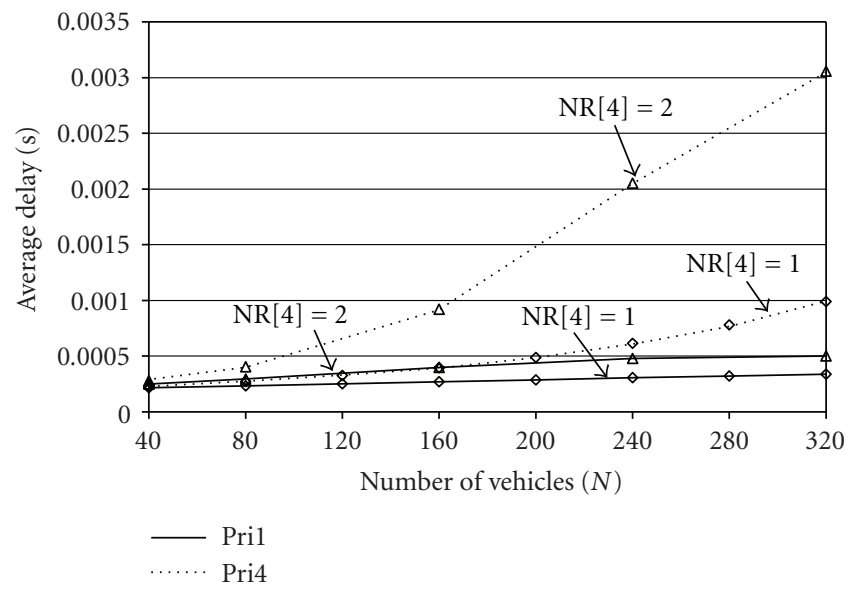

Figure 21: Average delay for each priority as a function of $\mathrm{N}$ and NR[4] for a system described in Tables 2 and 4.

reliability. This is due to the fact that as L increases, the traffic intensity increases, increasing the number of collisions and decreasing the reliability.

Figure 24 depicts the average delay versus $\mathrm{N}$ for different priorities and different values of L. From Figure 24, we conclude that larger the values of L, higher the average delay for both priority messages. Notice that for all cases the average delay is still acceptable, that is, it is lower than the message default lifetime.

5.2.4. Percentage of Pril Vehicles. In this subsection, we study the effect of the percentage of Pril vehicles, P1. Figures 25 and 26 depict the percentage of successful transmissions versus $\mathrm{N}$ for Pril and Pri4 messages, respectively, for different values of P1. We observe that as P1 increases there is a significant degradation in the percentage of successful transmissions, especially for large numbers of vehicles. This is due to the fact that as P1 increases, the traffic intensity of Pril messages increases, increasing the potential collisions, fact that decreases the percentage of successful transmissions.

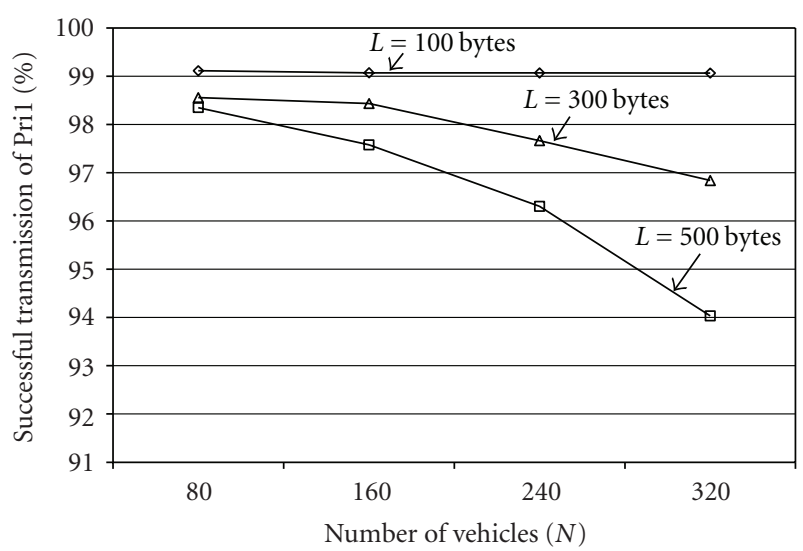

FIGURe 22: Percentage of Pril successful transmissions as a function of $\mathrm{N}$ and $\mathrm{L}$ for a system described in Tables 2 and 4.

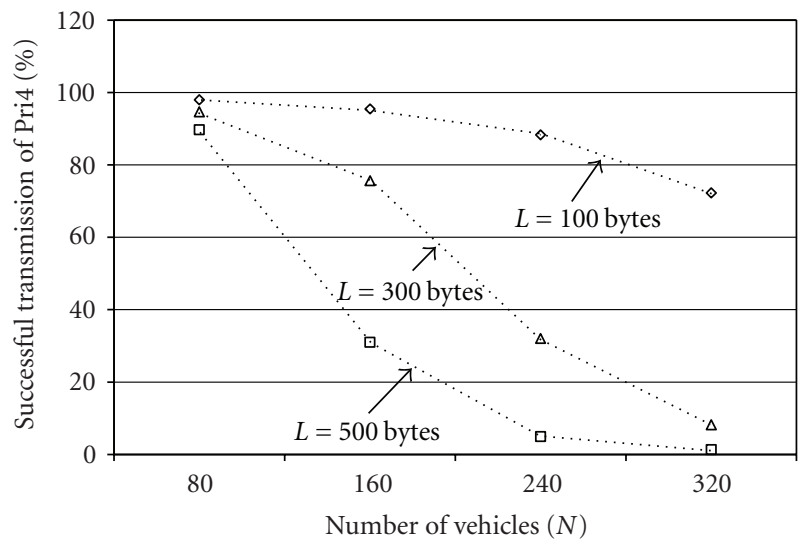

Figure 23: Percentage of Pri4 successful transmissions as a function of $\mathrm{N}$ and $\mathrm{L}$ for a system described in Tables 2 and 4.

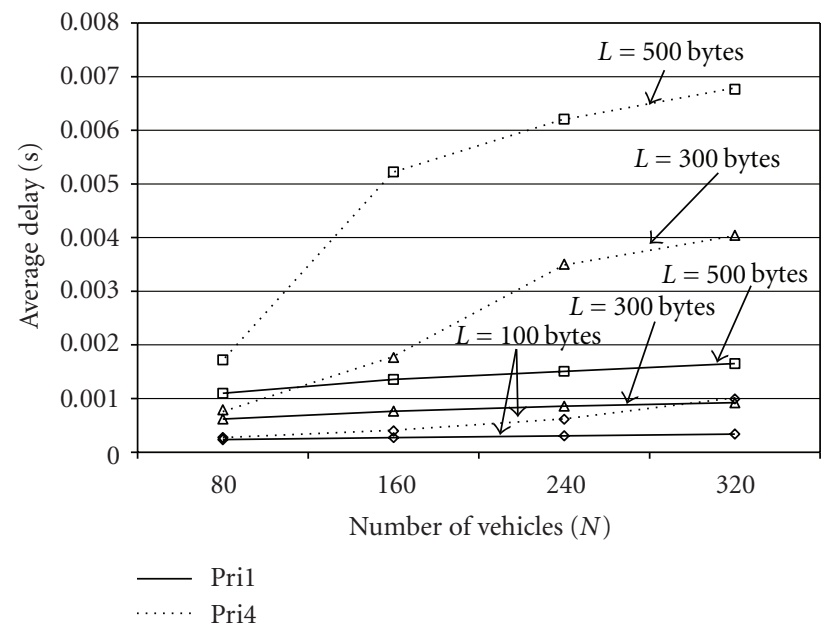

FIGURE 24: Average delay for each priority as a function of $\mathrm{N}$ and $\mathrm{L}$ for a system described in Tables 2 and 4 . 


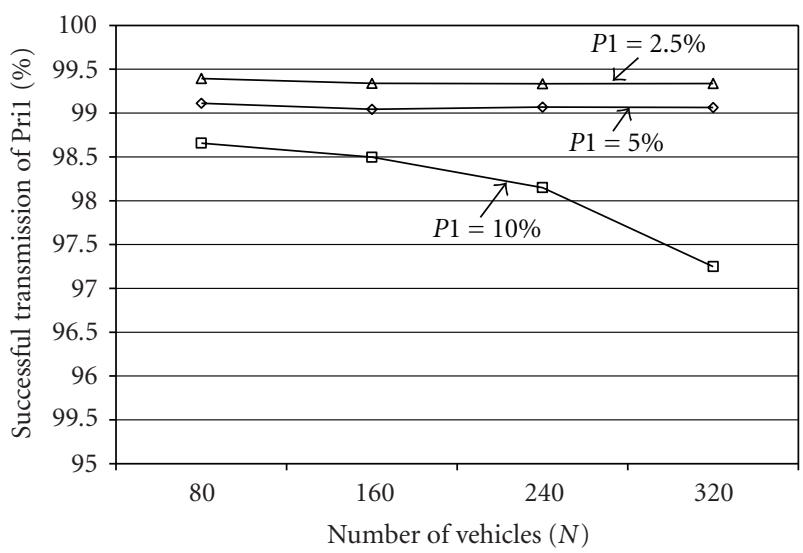

FIgURE 25: Percentage of Pril successful transmissions as a function of $\mathrm{N}$ and $\mathrm{P} 1$ for a system described in Tables 2 and 4 .

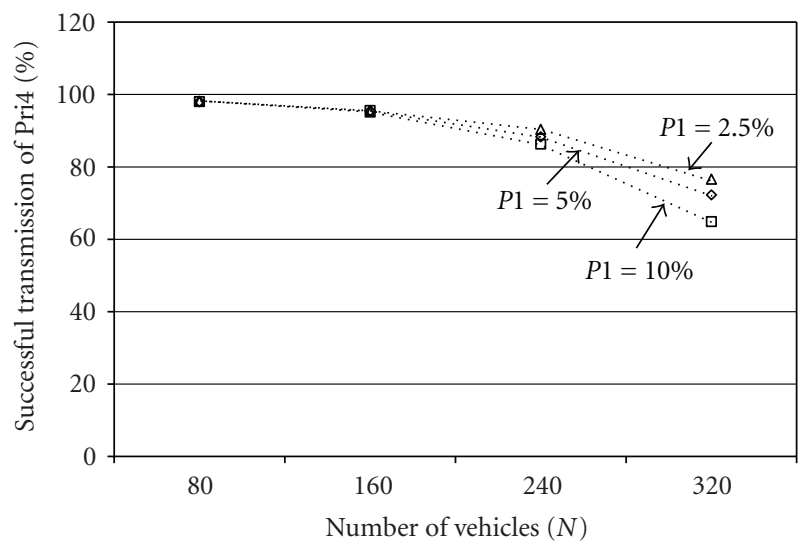

Figure 26: Percentage of Pri4 successful transmissions as a function of $\mathrm{N}$ and $\mathrm{P} 1$ for a system described in Tables 2 and 4 .

Figure 27 shows the average delay versus $\mathrm{N}$ for different priorities and different values of P1. Consistent with our observations concluded from Figures 25 and 26, we notice that for higher values of $\mathrm{P} 1$, the average delay increases for both priority messages. In all cases, the average delay is still acceptable, that is, it is lower than the message default lifetime.

\section{Performance Comparison between Vehicular Infrastructure and Ad hoc Networks}

In previous sections, we have shown the performance of our proposed protocol for different network architectures (infrastructure and ad hoc) versus different values of system parameters, such as number of vehicles, N, packet size, L, and percentage of Pril vehicles, P1. Moreover, each architecture uses unique parameters, such as RL[i] (for infrastructure) and NR[i] (for ad hoc). In this section, we aim to compare the performance of our proposed protocol in terms of Pril reliability in both vehicular infrastructure and vehicular ad hoc networks, as a function of the system parameters. We will not compare the protocols in terms of average delay as

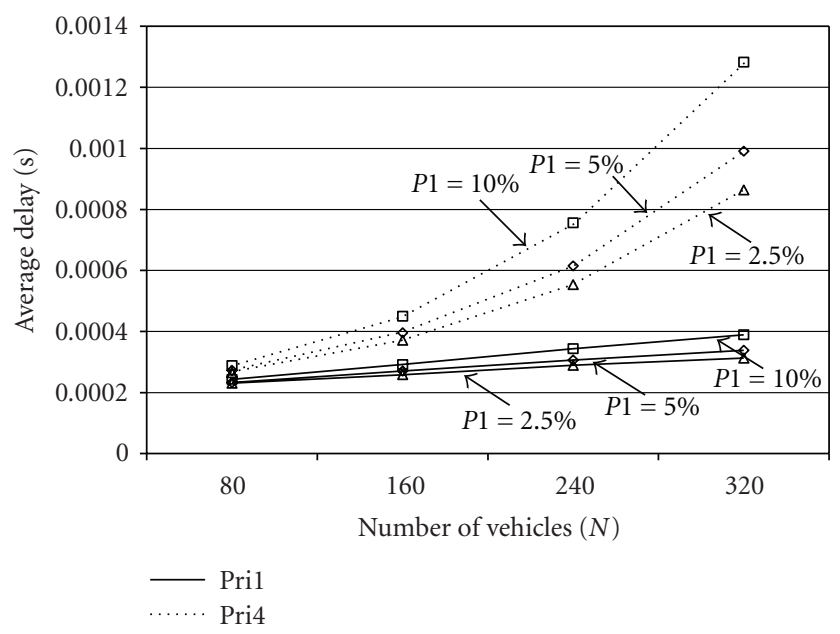

FIGURE 27: Average delay for each priority as a function of $\mathrm{N}$ and P1 for a system described in Tables 2 and 4 .

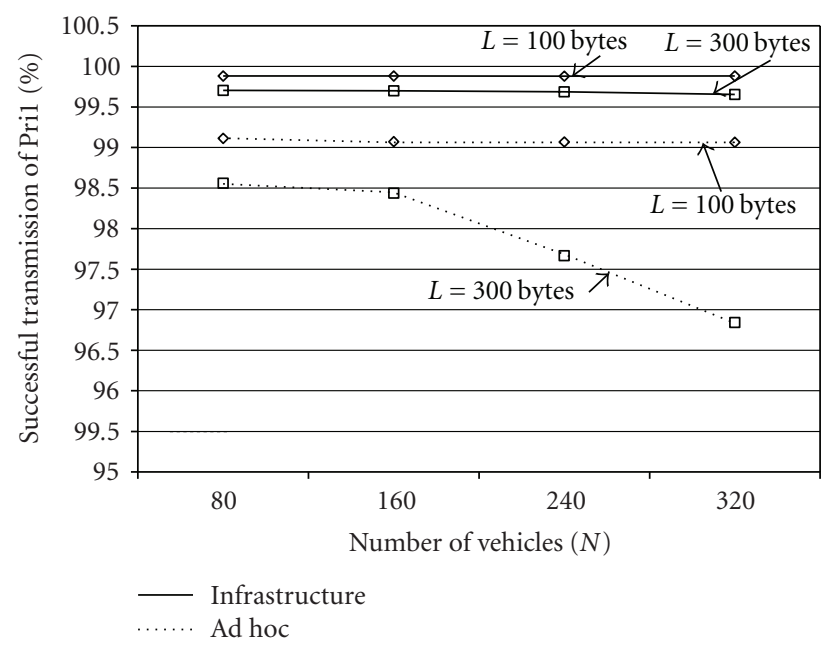

Figure 28: Comparison of percentage of Pril successful transmissions of both vehicular infrastructure and ad hoc networks as a function of $\mathrm{N}$ and $\mathrm{L}$ for a system described in Tables 2, 3 and 4.

the comparisons have already been shown in Sections 4 and 5. In all the cases the average delay is acceptable, that is, its value is below the default message lifetime.

6.1. Packet Size. In this subsection, we compare the performance of our proposed protocol in both networks as a function of L. Figure 28 shows the comparison of the percentage of Pril successful transmissions versus $\mathrm{N}$ for different values of $\mathrm{L}$ in both networks. We observe that the Pril performance of ad hoc networks is more sensitive to the values of $\mathrm{L}$ than the performance of infrastructure networks. This is due to the fact that in infrastructure networks we have an acknowledgement mechanism (from the RSU) that leads to reduced number of actual retransmissions. In ad hoc networks, on the other hand, the number of actual retransmissions is fixed, as determined by $\mathrm{NR}[i]$, leading to higher congestion and reduced reliability. 


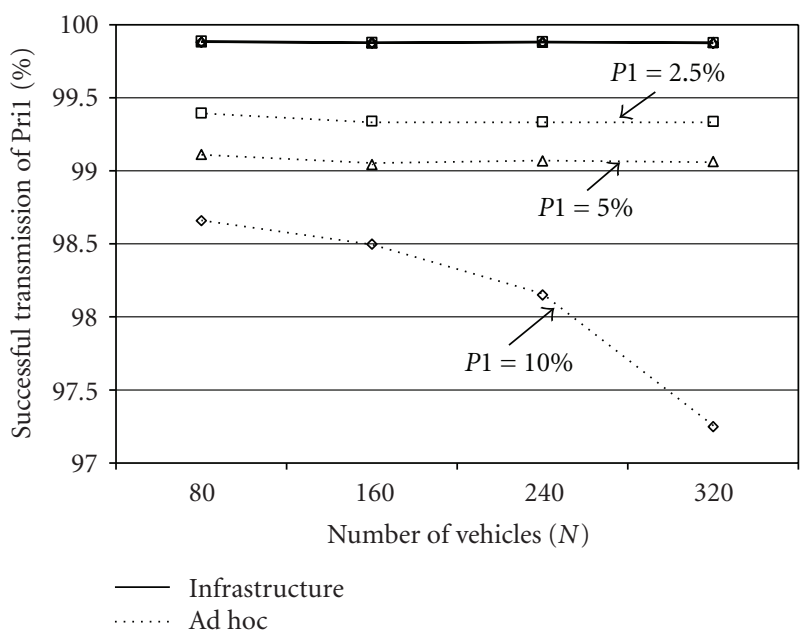

Figure 29: Comparison of percentage of Pril successful transmissions of both vehicular infrastructure and ad hoc networks as a function of $\mathrm{N}$ and $\mathrm{P} 1$ for a system described in Tables 2, 3 and 4.

6.2. Percentage of Pril Vehicles. Figure 29 shows the comparison of the percentage of Pril successful transmissions versus $\mathrm{N}$ for different values of $\mathrm{P} 1$ in both networks. We notice that as P1 increases, the reliability of Pril in infrastructure networks remains constant, but it decreases in ad hoc networks. This trend is especially pronounced for large values of $\mathrm{N}$.

As we observe from Figures 28 and 29 we notice that for all cases, the performance of an infrastructure network is superior to the ad hoc network. However, since the cost of the infrastructure is high, we should deploy it only in areas in which we can see a significant performance improvement that justifies our investment.

From Figure 29 we also observe that as the number of vehicles $\mathrm{N}$ increases, or the percentage of priority 1 increases, vehicular infrastructure networks have a significant performance advantage. Such system characteristics occur in urban areas where the potential number of vehicles and traffic accidents can be very high. Therefore, we recommend infrastructure-based IVC for urban areas where we can see a significant performance improvement that compensates for our deployment investment.

\section{Conclusions}

In this paper we propose to use IEEE 802.11e in conjunction with retransmissions to obtain priority-based communication in both infrastructure and ad hoc vehicular networks. The proposed protocol is fully compatible with both IEEE 802.11 and IEEE 802.11e standards. We have shown through OPNET-based simulations that the proposed protocol is a viable solution for priority-based intervehicle communication. Using proper settings of the proposed protocol parameters, $\mathrm{CW}_{\min }[i], \mathrm{CW}_{\max }[i]$, AIFS[i], TXOP[i], RL[i], and $\mathrm{NR}[i]$, our simulation results show that we can provide: (1) service differentiation for different message priorities, (2) very low average delays (well below the acceptable
$0.1 \mathrm{sec}$ for IVC), and (3) high percentage of successful transmissions (i.e., high reliability), even for large number of vehicles.

Based on the simulation results reported in the previous section, we infer that infrastructure-based IVC provides better performance for priority 1 messages in comparison to ad hoc IVC. The performance difference between the two network settings is more pronounced for large number of vehicles and high percentage of priority 1 messages (see Figure 29). In other words, infrastructure-based IVC will provide significant benefits in urban areas where the number of vehicles and potential traffic congestion are high. Therefore, for urban areas where we can see a significant performance advantage in the use of infrastructure networks, the monetary investment in such networks is justified. On the other hand, since the cost of an infrastructure-based network can be very high for rural areas, it may not be a necessity in those areas.

\section{References}

[1] International Road Traffic and Accident Database (IRTAD), "Road Traffic Data and Accident Traffic Data," June 2001.

[2] R. Bishop, "Intelligent Vehicle Application world-wide," IEEE Intelligent Systems, February 2000.

[3] D. Jones, "Keeping Cars from Crashing," IEEE Spectrum, Septenber 2001.

[4] European Transport Safety Council (ETSC), "Intelligent Transportation Systems and Road Safety Report," September 1999.

[5] Intelligent Transportation Systems (ITS) America, "IVI Candidate User Service," October 2001.

[6] Advanced Crusie-Assist Highway System Research Association (AHSRA) Japan, "Primary Requirement of Advanced CrusieAssist Highway System,” February 2001.

[7] M. Aoki, "Inetr-vehicle communication: technical issues on vehicle control application-oriented approach to ad-hoc networking," IEEE Communication Magazine, June 2001.

[8] A. Widodo and T. Hasegawa, "Impacts of environment adaptive running with and without IVCN," in Proceedings of the IEEE Vehicular Technology Conference (VTC'00), vol. 1, pp. 327-330, May 2000.

[9] M.-T. Sun, W. Feng, T. Lai, K. Yamada, H. Okada, and K. Fujimura, "GPS-based message broadcast for adaptive intervehicle communications," in Proceedings of the IEEE Vehicular Technology Conference (VTC '00), vol. 6, pp. 2685-2692, September 2000.

[10] Y. Morioka, T. Sota, and M. Nagakawa, "An anti-car collision system using GPS and $5.8 \mathrm{GHz}$ inter-vehicle communication at an off-sight intersection," in Proceedings of the IEEE Vehicular Technology Conference (VTC'00), September 2000.

[11] T. Nagaosa and T. Hasegawa, "Code assignment and the multicode sense scheme in an inter-vehicle CDMA communication network," IEICE Transactions on Fundamentals of Electronics, vol. E81-A, no. 11, pp. 2327-2333, 1998.

[12] Dedicated Short Range Communications Working Group, http://grouper.ieee.org/groups/802/11/Reports/tgp_update .htm.

[13] The Fleetnet Project, http://www.fleetnet.de.

[14] The Now: Network on Wheels Project, http://www.networkon-wheels.de/.

[15] Internet ITS Consortium, http://www.internetits.org/. 
[16] B. Williams and T. Camp, "Comparison of broadcasting techniques for mobile ad hoc networks," in Proceedings of the 3rd International Symposium on Mobile Ad Hoc Networking and Computing (MobiHOC '02), pp. 194-205, June 2002.

[17] D. Lee, R. Attias, A. Puri, R. Sengupta, S. Tripakis, and P. Varaiya, "A wireless token ring protocol for intelligent transportation systems," in Proceedings of the 4th IEEE Conference on Intelligent Transportation Systems (ITSC '01), pp. 11521157, August 2001

[18] K. Tokuda, M. Akiyama, and H. Fujii, "DOLPHIN for intervehicle communications system," in Proceedings of the IEEE Intelligent Vehicles Symposium (IV '00), pp. 504-509, October 2000.

[19] Q. Xu, T. Mak, J. Ko, and R. Sengupta, "Vehicle-to-vehicle safety messaging in DSRC," in Proceedings of the 1st ACM International Workshop on Vehicular Ad Hoc Networks (VANET '04), pp. 19-28, October 2004.

[20] Y. Kim and M. Nakagawa, "R-ALOHA protocol for SS inter-vehicle communication network using head spacing information," in Proceedings of the IEEE Intelligent Vehicles Symposium (IV'96), pp. 278-283, September 1996.

[21] F. Borgonovo, A. Capone, M. Cesana, and L. Fratta, "RRALOHA, a reliable R-ALOHA broadcast channel for ad-hoc inter-vehicle communication networks," in Proceedings of the 1st Annual Mediterranean Ad Hoc Networking Workshop (MedHoc-Net'02), September 2002.

[22] K. Shanmukh, G. Murthy, and R. Rao, "A decentralized Location-based Channel Access (LCA) protocol, particularly useful for inter vehicle communication," in Proceedings of the IEEE Vehicular Technology Conference (VTC '03), April 2003.

[23] M. Torrent-Moreno, D. Jiang, and H. Hartenstein, "Broadcast reception rates and effects of priority access in 802.11-based vehicular ad-hoc networks," in Proceedings of the 1st ACM International Workshop on Vehicular Ad Hoc Networks (VANET '04), pp. 10-18, October 2004.

[24] S. Yang, H. H. Refai, and X. Ma, "CSMA based inter-vehicle communication using distributed and polling coordination," in Proceedings of the 8th IEEE Conference on Intelligent Transportation Systems (ITSC '05), pp. 167-171, September 2005.

[25] C. Suthaputchakun and A. Ganz, "Military inter-vehicle communication with message priority using IEEE 802.11e," in Proceedings of the IEEE Military Communications Conference (MILCOM '06), October 2006.

[26] C. Suthaputchakun and A. Ganz, "Priority based intervehicle communication in vehicular ad hoc networks using IEEE802.11e," in Proceedings of the 65th IEEE Vehicular Technology Conference (VTC'07), April 2007.

[27] 802.11 WG, IEEE Std 802.11e/D10.0, "Draft Amendment to Standard [for] Information TechnologyTelecommunications and Information Exchange Between Systems-LAN/MAN Specific Requirements-Part 11: Wireless Medium Access Control (MAC) and Physical Layer (PHY) Specifications: Medium Access Control (MAC) Quality of Service (QoS) Enhancements," September 2004.

[28] OPNET Technologies, Inc., "OPNET Modeler v11.5," http://www.opnet.com.

[29] H. Daizo, T. Iwahashi, M. Bandai, and T. Watanabe, "An intervehicle communication MAC protocol supported by road side communication and its extension," in Proceedings of the 1st ACM International Workshop on Vehicular Ad Hoc Networks (VANET'04), October 2004.

[30] G. Korkmaz and E. Ekici, "Urban multi-hop broadcast protocol for inter-vehicle communication systems," in Proceedings of the 1st ACM International Workshop on Vehicular Ad Hoc Networks (VANET'04), pp. 76-85, October 2004.

[31] K. Fujimura and T. Hasegawa, "A collaborative MAC protocol for inter-vehicle and road to vehicle communications," in Proceedings of the 7th IEEE Conference on Intelligent Transportation Systems (ITSC '04), pp. 816-821, October 2004.

[32] M. Lott, "Performance of a medium access scheme for intervehicle communication," in Proceedings of the International Symposium on Performance Evaluation of Computer and Telecommunication Systems (SPECTS '02), July 2002. 

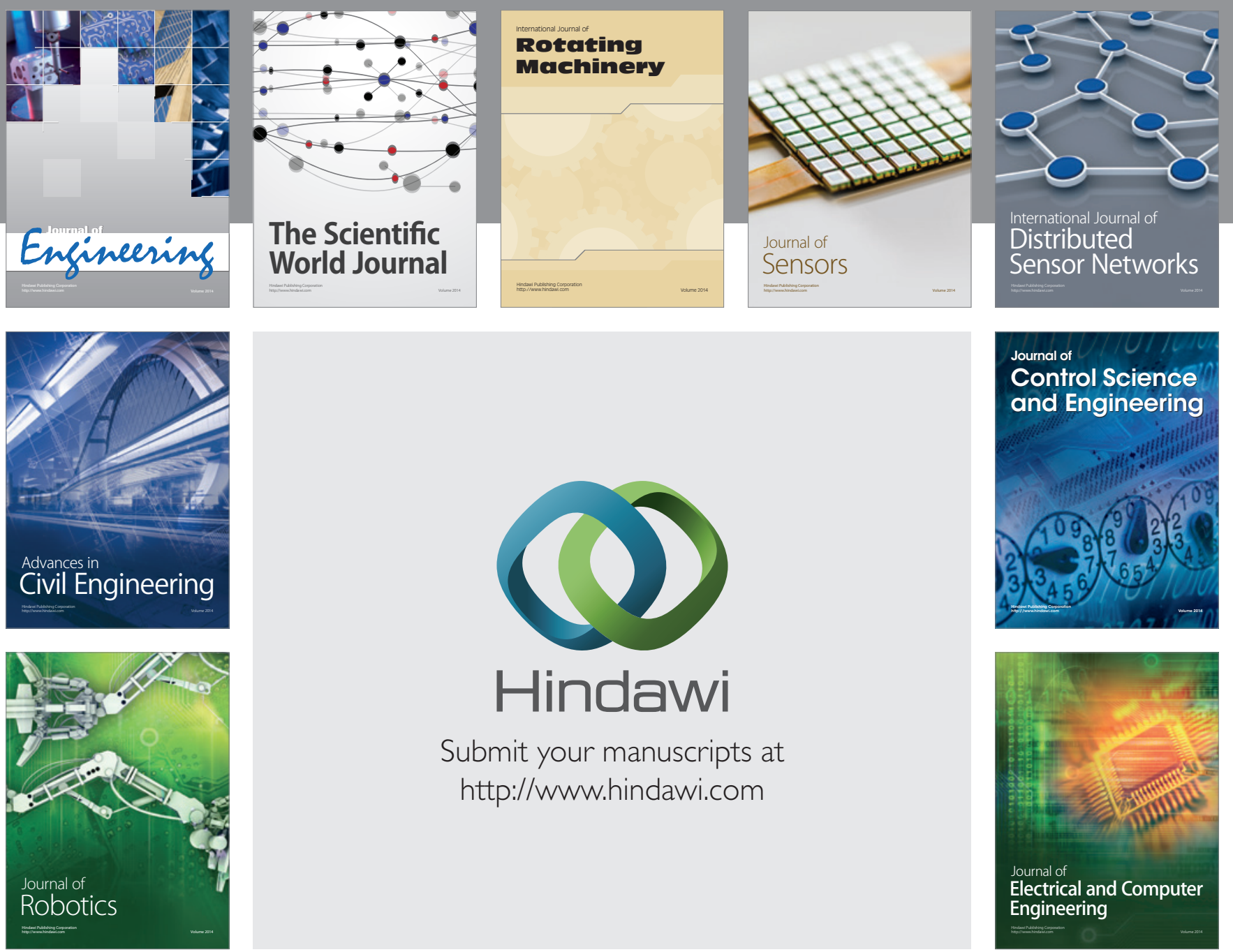

Submit your manuscripts at

http://www.hindawi.com
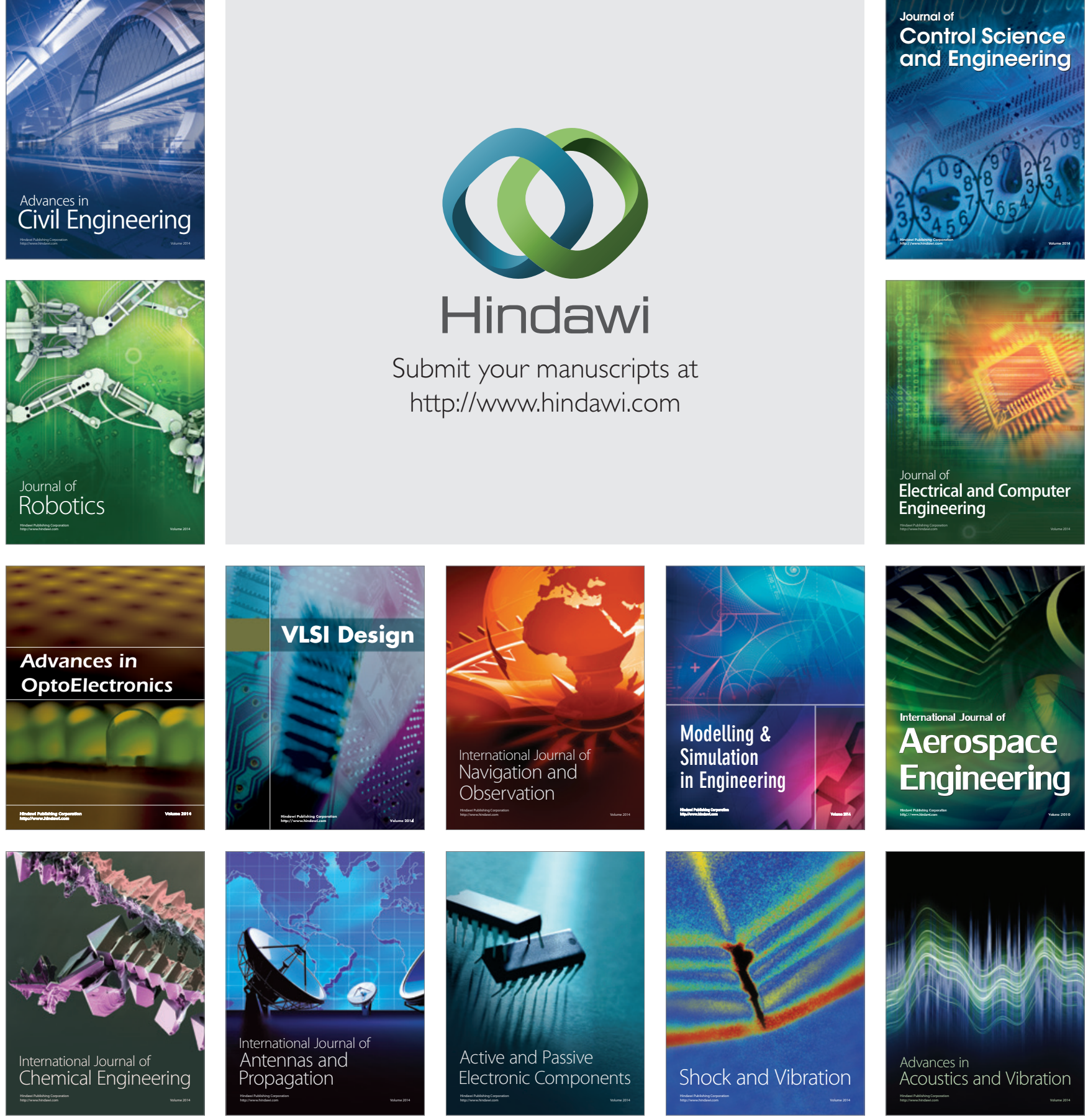